\title{
POSTER
}

\section{A propos d'un cas de carcinome verruqueux}

\section{Tong S, Grenier S, Nicolas C, Lefevre B, Hafian H, Laurence S}

Service d'odontologie - CHU de REIMS

Le carcinome verruqueux forme au niveau buccal une lésion leucoplasique, exophitique, papillomateuse ou verruqueuse localement agressive (Spiro, 1998). Cette pathologie représente 2 à $12 \%$ de l'ensemble des carcinomes de la cavité buccale, avec un âge moyen de découverte de 69 ans et une prédilection masculine. L'intoxication tabagique et la mauvaise hygiène buccodentaires sont incriminées dans la genèse de ce carcinome (Koch, 2001), ainsi que l'infection à HPV 16 et 18 retrouvée dans plus de $40 \%$ des carcinomes verruqueux de la cavité orale (Barnes, 2005). Les localisations buccales préférentielles sont la face interne des joues et la gencive (Salesiotis, 2003).

Le cas rapporté est celui d'une patiente de 78 ans adressée par son dentiste qui a diagnostiqué une kératose douloureuse, localisée dans le secteur maxillaire gauche et pour laquelle une origine prothétique est suspectée. Les antécédents médicaux comportent un diabète de type 2 traité et équilibré, ainsi qu'une HTA contrôlée sous traitements. L'anamnèse relate une lésion blanche située sur l'arcade maxillaire gauche présente depuis plus d'une année et à l'origine d'une gêne notamment lors des repas. L'examen exobuccal est sans particularité. L'examen endobuccal met en évidence un édentement partiel compensé au maxillaire par une prothèse adjointe mal adaptée et traumatisante. La mandibule n'est pas appareillée. L'hygiène buccodentaire est défaillante, avec la présence de tartre et de plaque sur les dents restantes, dans un contexte de parodontite chronique généralisée. Au niveau de la zone tubérositaire maxillaire gauche, débordant sur la voûte palatine et dans le fond du vestibule, une lésion kératosique, inhomogène et villeuse mesurant environ $2 \mathrm{~cm}$ dans son grand axe est objectivée. La palpation de la lésion est douloureuse. L'orthopantomogramme ne révèle pas d'anomalie hormis une lyse osseuse en rapport avec la parodontite. Une biopsie de la lésion est pratiquée et le port de la prothèse est proscrit. L'analyse histologique conclut à une acanthose avec papillomatose et hyperkératose sans caractère atypique de nature réactionnelle. Lors du contrôle un mois plus tard, la lésion n'a pas régressé malgré l'éviction du facteur traumatique. L'aspect inhomogène, chevelu et la persistance de la lésion conduisent à son exérèse complète avec mise en place d'une plaque palatine. L'analyse histologique de la pièce opératoire conclut à un carcinome verruqueux avec des limites d'exérèse saines. Après contrôle de cicatrisation et annonce du diagnostic, un suivi régulier est instauré. Cependant la patiente ne peut se rendre à ses visites de contrôle suite à une chute. Elle n'est examinée qu'un an après l'exérèse et une lésion kératosique est de nouveau observée sur le site initial. Son analyse histologique après ablation révèle une dysplasie malpighienne de grade modéré.

Ce cas exposé révèle la difficulté du diagnostic du carcinome verruqueux, dont l'analyse histologique nécessite des pièces d'exérèse larges et profondes. Le traitement chirurgical est le traitement de choix associé à un suivi, pour éliminer précocement les récidives fréquentes dans ce type de pathologie.

This is an Open Access article distributed under the terms of the Creative Commons Attribution License 4.0, which permits unrestricted use, distribution, and reproduction in any medium, provided the original work is properly cited. Article disponible sur le site nttp://Www.Stco-congres.org ou nttp://dx.dol.org/10.1051/stco/20166403020 